\title{
FUS/TLS forms cytoplasmic aggregates, inhibits cell growth and interacts with TDP-43 in a yeast model of amyotrophic lateral sclerosis
}

\author{
Dmitry Kryndushkin ${ }^{1,2}$, Reed B. Wickner ${ }^{1}$, Frank Shewmaker ${ }^{2}$ \\ ${ }^{1}$ Laboratory of Biochemistry and Genetics, National Institute of Diabetes Digestive and Kidney Diseases, National Institutes of \\ Health, Bethesda, MD 20892, USA \\ 2 Department of Pharmacology, Uniformed Services University of the Health Sciences, Bethesda, MD 20814, USA \\ $凶$ Correspondence: frank.shewmaker@gmail.com \\ Received February 28, 2011 Accepted March 6, 2011
}

\begin{abstract}
Amyotrophic lateral sclerosis (ALS) is a fatal disease characterized by the premature loss of motor neurons. While the underlying cellular mechanisms of neuron degeneration are unknown, the cytoplasmic aggregation of several proteins is associated with sporadic and familial forms of the disease. Both wild-type and mutant forms of the RNA-binding proteins FUS and TDP-43 accumulate in cytoplasmic inclusions in the neurons of ALS patients. It is not known if these so-called proteinopathies are due to a loss of function or a gain of toxicity resulting from the formation of cytoplasmic aggregates. Here we present a model of FUS toxicity using the yeast Saccharomyces cerevisiae in which toxicity is associated with greater expression and accumulation of FUS in cytoplasmic aggregates. We find that FUS and TDP-43 have a high propensity for co-aggregation, unlike the aggregation patterns of several other aggregation-prone proteins. Moreover, the biophysical properties of FUS aggregates in yeast are distinctly different from many amyloidogenic proteins, suggesting they are not composed of amyloid.
\end{abstract}

KEYWORDS amyotrophic lateral sclerosis (ALS), fused in sarcoma (FUS), TLS, proteinopathy, yeast

\section{INTRODUCTION}

Amyotrophic lateral sclerosis (ALS) is a debilitating and fatal neurodegenerative disease that is linked to the selective degeneration of motor neurons. The disease typically manifests in midlife and symptoms include progressive muscle weakness with eventual paralysis; death usually occurs from respiratory failure approximately three years after symptom onset. Most cases of ALS are apparently sporadic, but about $10 \%$ are familial, and causative mutations have been identified in the SOD1, TARDBP and FUS/TLS genes. In each case the protein product of the gene is observed to form aberrant cytoplasmic inclusions in the diseased neurons (Rosen et al., 1993; Sreedharan et al., 2008; Kwiatkowski et al., 2009; Vance et al., 2009). Despite much focus on these disease-linked genes, a mechanistic explanation that links motor neuron degeneration and cytoplasmic protein aggregation remains elusive.

The RNA-binding proteins FUS (fused in sarcoma) and TDP-43 (product of TARDBP gene) have each been connected to RNA processing and control (reviewed (Lagier-Tourenne et al., 2010)). These proteins are similar in having RNA-recognition motifs and glycine-rich domains, and they both normally primarily localize to the nucleus, but also have been shown to shuttle between the nucleus and cytoplasm (Zinszner et al., 1997; Ayala et al., 2008). Moreover, there is evidence that they are involved in the formation of stress granules (Colombrita et al., 2009; Bosco et al., 2010; Gal et al., 2010; Ito et al., 2011). It is not known if their cytoplasmic accumulation results in a loss-of-function toxicity or if the cytoplasmic inclusions themselves introduce a new toxicity, or possibly both. In the case of TDP-43, various studies have not definitively shown whether increased levels, decreased function or aggregated TDP-43 is the driver of pathogenesis. In the case of FUS, there is little data due to a paucity of model systems.

The yeast Saccharomyces cerevisiae is an established 
model for studying the cellular mechanisms of toxicity that are associated with protein misfolding and aggregation in neurodegenerative diseases (Bharadwaj et al., 2010; Braun et al., 2010; Khurana and Lindquist, 2010). Yeast contain native proteins that can aggregate and produce toxicity (McGlinchey et al., 2011), and have mechanisms that are conserved among eukaryotes to efficiently cope with protein misfolding and aggregation. A general research strategy, using yeast cells, involves the expression of various diseaseassociated and aggregation-prone proteins whose cellular accumulation is specifically linked to disease. A huntingtin fragment with expanded polyglutamine tract (Meriin et al., 2002; Duennwald and Lindquist, 2008), a-synuclein (Outeiro and Lindquist, 2003; Sharma et al., 2006) and TDP-43 (Johnson et al., 2008)—all produce strong toxicity concurrent with increased expression in yeast cells. Furthermore, unbiased genetic screens employing these proteins have successfully identified proteins that can either enhance or reduce the induced toxicity. Importantly, the modifiers that resulted from such screens were not generally overlapping; indicating that the toxicity produced by these proteins may be attributed to their specific biological functions, and not related to a general aggregation phenomenon. Therefore, the approach of yeast expression for disease-associated proteins may lead to the discovery of specific cellular pathways counteracting toxicity.

Here, we examine the expression of human FUS and TDP-43 in yeast, which has no homologues of these proteins. We show that yeast can serve as a FUS/TLS proteinopathy model suitable to investigate the mechanisms of FUS aggregation and toxicity on the cellular level. Information gained from a yeast model should be applicable to mammalian neuronal culture, because cellular pathways that govern protein misfolding are highly conserved among eukaryotes (Cooper et al., 2006; Yeger-Lotem et al., 2009). Additionally, we investigate the localization and character of both FUS and TDP-43 aggregates and find that they recapitulate many of the observations made in diseased tissues.

\section{RESULTS}

\section{FUS expression in yeast cells results in rapid aggregation and toxicity}

In order to get insights into the mechanisms of FUS toxicity and aggregation, we first utilized a galactose inducible promoter to achieve high-level expression of full-length FUS in yeast cells. To track cellular localization of FUS during expression, a FUS-GFP fusion was made and expressed in parallel with the untagged version. Cells were grown to log phase under non-inducing conditions (in raffinose medium) and then were plated on galactose medium to induce expression. Both FUS and FUS-GFP similarly caused severe cell growth inhibition during expression (Fig. 1A). Such inhibition was previously reported for TDP-43 (Johnson et al., 2008), which likewise inhibited growth using our expression system (Fig. 1A). For additional comparison, also

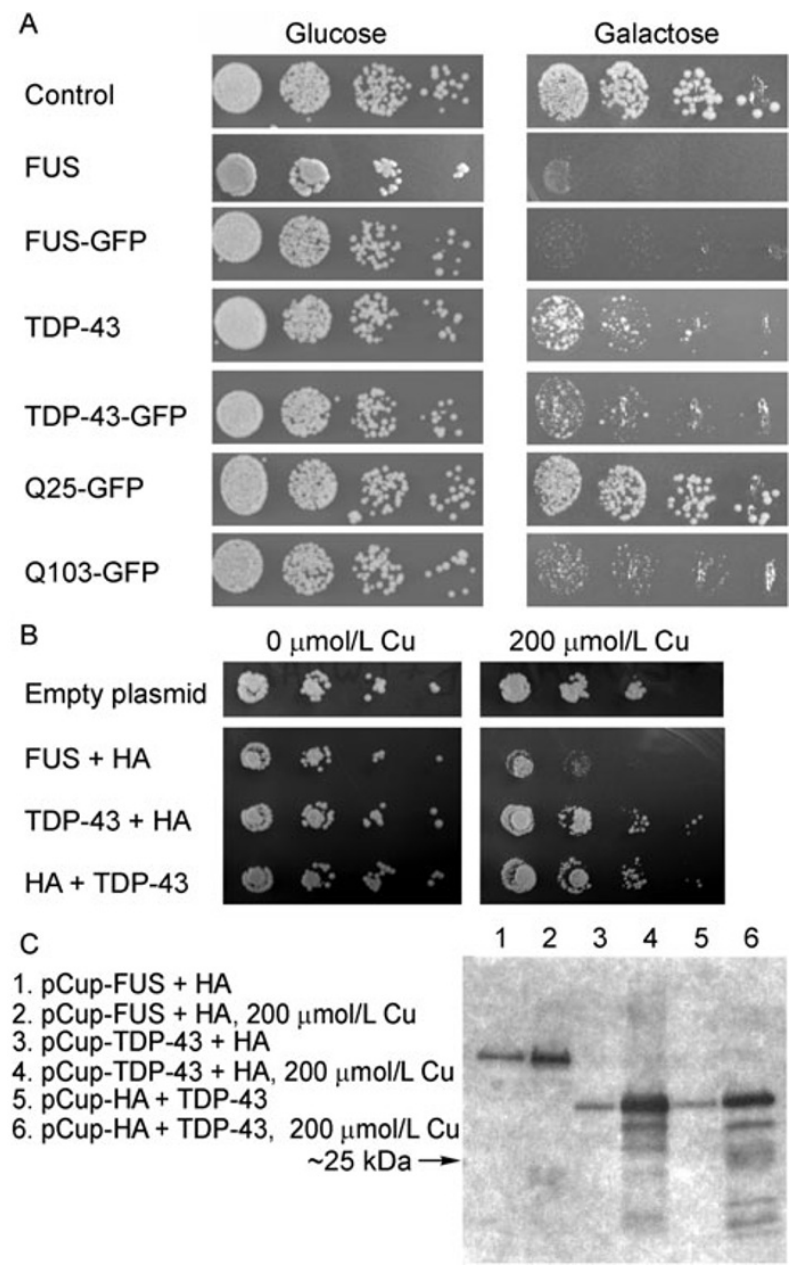

Figure 1. Relative toxicity of FUS, TDP-43 and polyglutamine in yeast. (A) Galactose-inducible (GAL1 promoter) expression vectors were used to compare the relative effects of expression of various FUS, TDP-43 and polyglutamine constructs (HttQ25 and HttQ103) in Saccharomyces cerevisiae strain BY4741. Cells were grown in SDRaf to mid-log phase; then $5 \times$ serial dilutions were spotted on $2 \%$ glucose or $2 \%$ galactose $/ 1 \%$ raffinose SD agar plates and grown for three days. Co-expression of FUS and TDP-43 in the same cells resulted in severe toxicity that was equal to, but not obviously greater than, expression of FUS alone (data not shown). (B) Hemagglutinin A (HA) epitope-tagged FUS and TDP-43 were expressed in strain BY4741 under the control of a copperinducible promoter; $10 \times$ serial dilutions were spotted on SD agar plates with or without copper. (C) A western blot using anti-HA antibody was performed on equivalent cell lysates from copper-induced and un-induced cells. Placing an HA tag at either the amino-terminal or C-terminal end of TDP-43 had no obvious differential affect on expression. 
expressed was HttQ103-GFP (N-terminal polyglutamineexpanded fragment of the human Huntingtin protein), which was shown previously to cause glutamine length-dependent toxicity in yeast via suppression of endocytosis (Meriin et al., 2003). For control, the non-pathological human Huntingtin fragment (HttQ25) was also included.

All constructs were expressed under identical conditions using the galactose inducible GAL1 promoter to allow synchronous induction of protein expression in all cells. All three proteins clearly caused toxicity, with FUS and FUS-GFP exhibiting the strongest effect. Milder toxicities could be achieved using a copper-inducible promoter (CUP1) that, in our experience, produces less expression than the galactoseinducible promoter (Fig. 1B). The different toxicities exhibited by FUS and TDP-43 do not appear to be a result of differential protein expression (Fig. 1C).

The distribution and localization of expressed FUS-GFP and TDP-43-GFP were visualized by fluorescent microscopy $(2 \mathrm{~A})$. Even at the very earliest time points during galactose induction (20 min), FUS-GFP displayed abundant aggregation (several foci per cell) (Fig. 2A). Note that the kinetics of TDP-43-GFP aggregation was slower: the first foci or focus can be detected only after $40-50 \mathrm{~min}$ of induction. To estimate aggregation propensity of FUS during yeast expression, we utilized the CUP1 inducible promoter to achieve low-tomoderate FUS expression levels. However, even in the absence of induction (without $\mathrm{Cu}$ ) we still observed FUS cytoplasmic foci in approximately $50 \%$ of cells (Fig. 2B and 3D). That basal expression from the copper promoter still resulted in FUS cytoplasmic aggregates suggests that FUS has a high intrinsic propensity for aggregation in yeast, which is consistent with previous observations (Fushimi et al., 2011).

\section{The whole sequence of FUS is required for toxicity and aggregation}

FUS is a 526 -amino acid, multi-domain protein that possesses several C-terminal elements indicative of RNA binding and an $\mathrm{N}$-terminal half that is highly enriched in serine, glutamine, tyrosine and glycine (Fig. 3A) (Iko et al., 2004). We generated several truncation constructs to dissect determinants of FUS toxicity in yeast. We expressed each of the truncated FUS constructs using a galactose-inducible promoter (Fig. 3B) and a copper-inducible promoter (data not shown). Strong growth inhibition was only observed when fulllength FUS was expressed. Removal of segments from either the $\mathrm{C}$-terminal or the $\mathrm{N}$-terminal domains abrogated toxicity. Two FUS constructs were constructed with the HA epitope tag, which had no effect on the toxicity of the constructs after galactose induction (Fig. 3B, lower panel). Both constructs had similar levels of expression (Fig. $3 C$ ), illustrating uniform induction with the GAL1 promoter. The observed toxicity of full-length FUS is consistent with recent findings from the $\mathrm{Wu}$

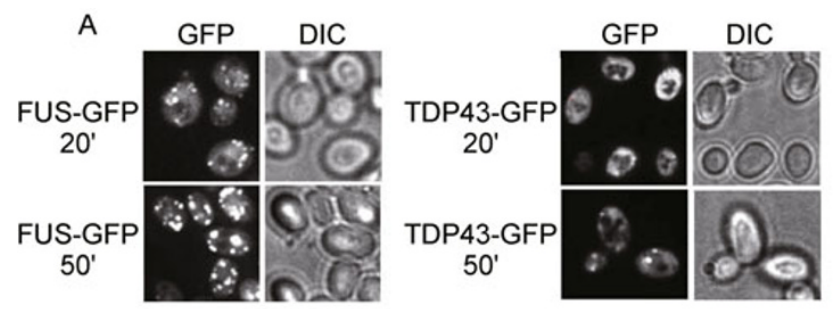

B

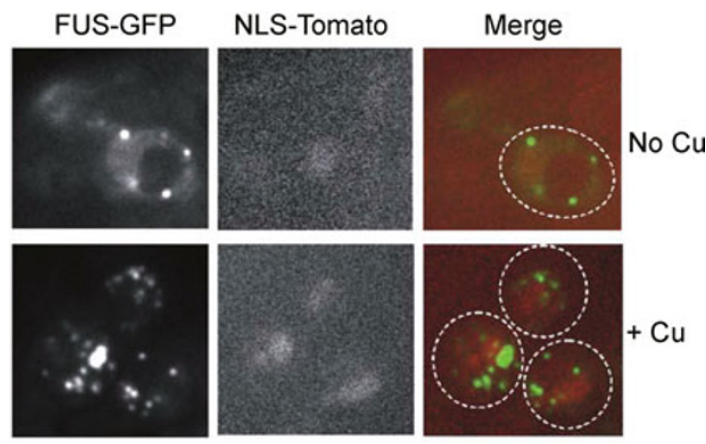

C

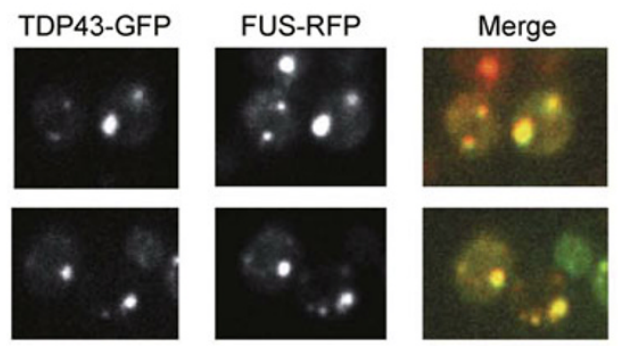

D

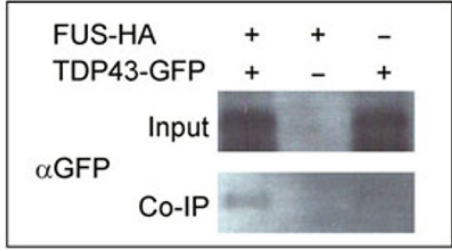

Figure 2. FUS and TDP-43 aggregate and co-localize in cytoplasmic inclusions. (A) Expression of the GAL1controlled FUS and TDP-43 GFP fusions were induced and the speeds of aggregate formation were compared by fluorescence microscopy. (B) The co-expression of a nuclearlocalized marker (NLS-tomato) indicates that FUS-GFP inclusions are cytoplasmic, even at lower expression levels. (C) Coexpression of the GAL1-controlled FUS-mRFP and TDP-43GFP results in co-localized aggregates as observed by confocal microscopy. (D) FUS and TDP-43 co-immunoprecipitate. Lysates were prepared of cells expressing FUS-HA, TDP-43-GFP or both. FUS-HA was pulled-down with anti-HAconjugated agarose and subsequent western blots were performed by probing against TDP-43-GFP.

laboratory (Fushimi et al., 2011). Moreover, they found that FUS expression could result in actual cell death, indicating that toxicity is not limited to growth inhibition. 


\section{A FUS/TLS}

\begin{tabular}{|l|l|l|l|l|l|l|l|}
\multicolumn{1}{|c|}{165} & \multicolumn{2}{c}{288} & 366 & 426 & 449 & 526 \\
\hline SQGY & RGG & RRM & RGG ZnF & RGG \\
\hline
\end{tabular}

B

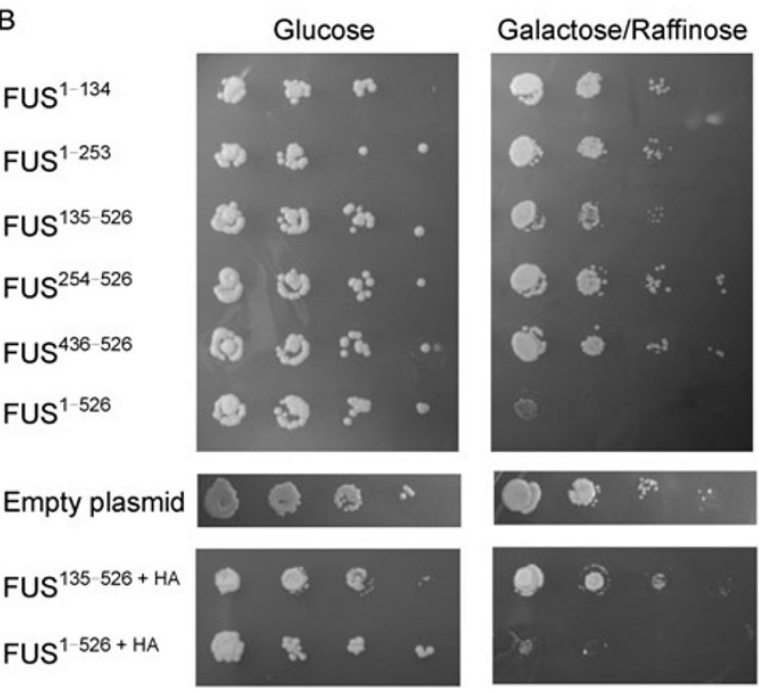

C
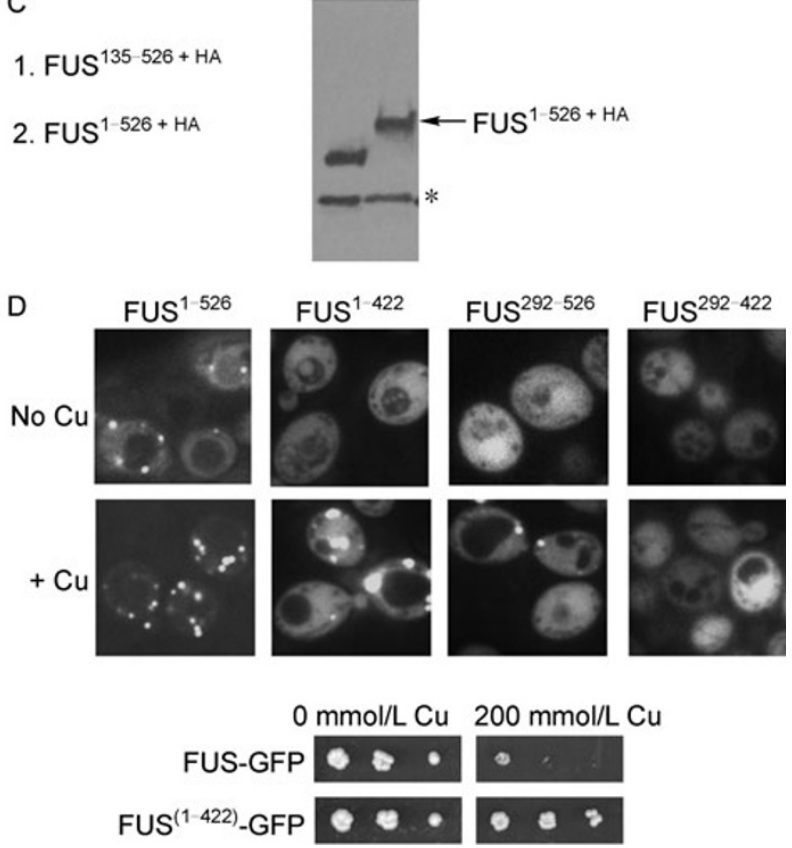

Figure 3. The full-length FUS protein is most aggregation-prone and toxic in yeast. (A) Schematic picture of the full-length FUS protein as characterized by lko and coworkers (lko et al., 2004). SQGY denotes the region that is highly enriched for serine, glutamine, glycine and tyrosine; RGG, rich in arginine and/or glycine; RRM, RNA recognition motif; ZnF, zinc finger domain. (B) Strain BY4741 harboring a series of GAL1-controlled FUS truncations was grown on both SD and SDGal plates as described in Fig. 1A, using $10 \times$ serial dilutions. (C) A western blot with anti-HA antibody was performed on equivalent cell lysates after 6 hours of galactose induction, indicating that full-length FUS toxicity was not due to greater protein expression; *, degradation product. (D) Fluorescence microscopy was used to observe GFP fused to full-length and truncated FUS under the control of a copper-inducible promoter (upper panel). The aggregation of FUS ${ }^{(1-422)}$-GFP did not correspond with an inhibition of cell growth (lower panel). 
A reduction in toxicity was coupled with diminished aggregation potential of truncated FUS constructs. Most evident was the absence of aggregation of FUS deletion mutants expressed under control of the CUP1 promoter in the absence of induction (e.g., without $\mathrm{Cu}$ ), while under the same conditions full-length FUS still formed fluorescent foci (Fig. 3D). The fluorescence intensities of all FUS-GFP constructs were similar, confirming similarity in their expression levels. After copper induction, both FUS ${ }^{(1-422)}$-GFP and FUS ${ }^{(292-526)}$-GFP were able to form aggregates; however, the formation of foci and toxicity was still significantly reduced compared to full-length FUS (Fig. 3D).

\section{FUS and TDP-43 co-localize and interact in yeast}

In neurons, both FUS and TDP-43 predominantly localize to cell nuclei under physiological conditions, but both proteins were found to co-localize in cytoplasmic inclusions in the spinal motor neurons of ALS patients (Deng et al., 2010). Coexpression of FUS-RFP and TDP-43-GFP in yeast cells resulted in cytoplasmic aggregates that unambiguously contained both proteins, as evident from confocal fluorescent microscopy (Fig. 2C). Remarkably, perfect colocalization was observed in every single cell that contained both FUS-RFP and TDP-43-GFP visible foci, indicating a strong interaction between the two proteins. It has been proposed that FUS and TDP-43 functionally interact as part of a larger biochemical complex in mammalian cells (Kim et al., 2010), and they have been shown to co-immunoprecipitate when expressed in cultured cells (Kim et al., 2010; Ling et al., 2010). We likewise found that TDP-43 specifically co-immunoprecipitated with FUS in yeast cell extracts, when both proteins were expressed (Fig. 2D).

FUS aggregates do not localize to the same compartment as many amyloidogenic proteins, but do co-localize with polyglutamine

It was previously reported that mammalian and yeast cells share common protein quality control mechanisms, with similar patterns of sequestration and localization of misfolded proteins (Kaganovich et al., 2008). Many amyloidogenic proteins were reported to be localized to a single "insoluble protein deposit," or IPOD, a putative compartmentalization of terminally-aggregated proteins that have exceeded other mechanisms of protein quality control. In yeast cells with expressed FUS, we did not observe localization to the nucleus, but instead extensive cytoplasmic inclusions were formed even at low expression levels. The localization of these aggregates was compared with other aggregationprone proteins that were previously shown to localize to the IPOD. FUS aggregates did not co-localize with several described IPOD markers (Kaganovich et al., 2008), such as Hsp104-GFP, Ubc9ts-GFP and aggregates of overproduced amyloidogenic proteins Ure2-GFP or RNQ-GFP (Fig. 4A). In addition, no co-localization was observed with $\alpha$-synucleinGFP (another amyloid-related protein forming toxic aggregates in yeast cells (Outeiro and Lindquist, 2003)) (Fig. 4A). However, FUS-GFP did partially co-localize with overproduced huntingtin fragment containing an extended polyglutamine track, HttQ103-GFP (Fig. 4B). This is remarkable because FUS was previously found to be a major component of neuronal intranuclear polyglutamine inclusions of Huntington's disease (Woulfe et al., 2010). It is striking that while FUS-GFP did not co-localize with many other misfolded proteins, it did show co-localization with TDP-43 (Fig. 2C) and huntingtin fragment, indicating strong specificity of this interaction.

\section{FUS inclusions are detergent sensitive and differ from amyloid aggregates}

Saccharomyces cerevisiae has several native proteins that can adopt an infectious, or prion, state that is based on a selfpropagating amyloid conformation (Wickner et al., 2010). The Hsp104p chaperone is required for the propagation of these amyloid-based prions and is believed to act by fragmenting the infectious amyloid fibers, thus creating new prion "seeds" and facilitating proliferation of the prion. Deletion of HSP104, or inhibition of Hsp104p, results in the formation of very large aggregates and finally leads to rapid prion loss during cellular divisions (Chernoff et al., 1995; Kryndushkin et al., 2003). Likewise, Hsp104p is necessary for the manifestation of HttQ103 (Krobitsch and Lindquist, 2000; Meriin et al., 2002), but not TDP-43 (Johnson et al., 2008) aggregation and toxicity. We checked if Hsp104p would similarly influence FUS toxicity and found that deletion of the HSP104 gene neither changed the FUS aggregation pattern (data not shown), nor eliminated FUS toxicity (Fig. 5).

Amyloid-based yeast prions and proteins with polyglutamine (polyQ) expansions share extreme resistance to treatments with strong anionic detergents, such as sodium dodecyl sulphate (SDS) or sarcosyl (Serio et al., 2000; Speransky et al., 2001; Kryndushkin et al., 2003; Salnikova et al., 2005). This reflects a tight packing of $\beta$-sheets within the amyloid backbone, making it a characteristic feature of amyloid structure. We tested whether the FUS aggregates that form in yeast have similar properties. Yeast cells expressing FUS-GFP protein for 4 hours were harvested, disrupted and analyzed by simple sedimentation assay (centrifugation 16,000 $\mathrm{g}$ for $10 \mathrm{~min}$ ). In parallel, Triton X-100 and RNase $A$ were applied to cell extracts for 10 min followed by the same spin. About $70 \%$ of FUS-GFP protein was found in the pellet fractions independent of treatments (Fig. 6A), indicating the presence of FUS in very high molecular weight complexes that are not dependent on membrane associations.

Aggregation behavior of FUS was compared with TDP-43, 


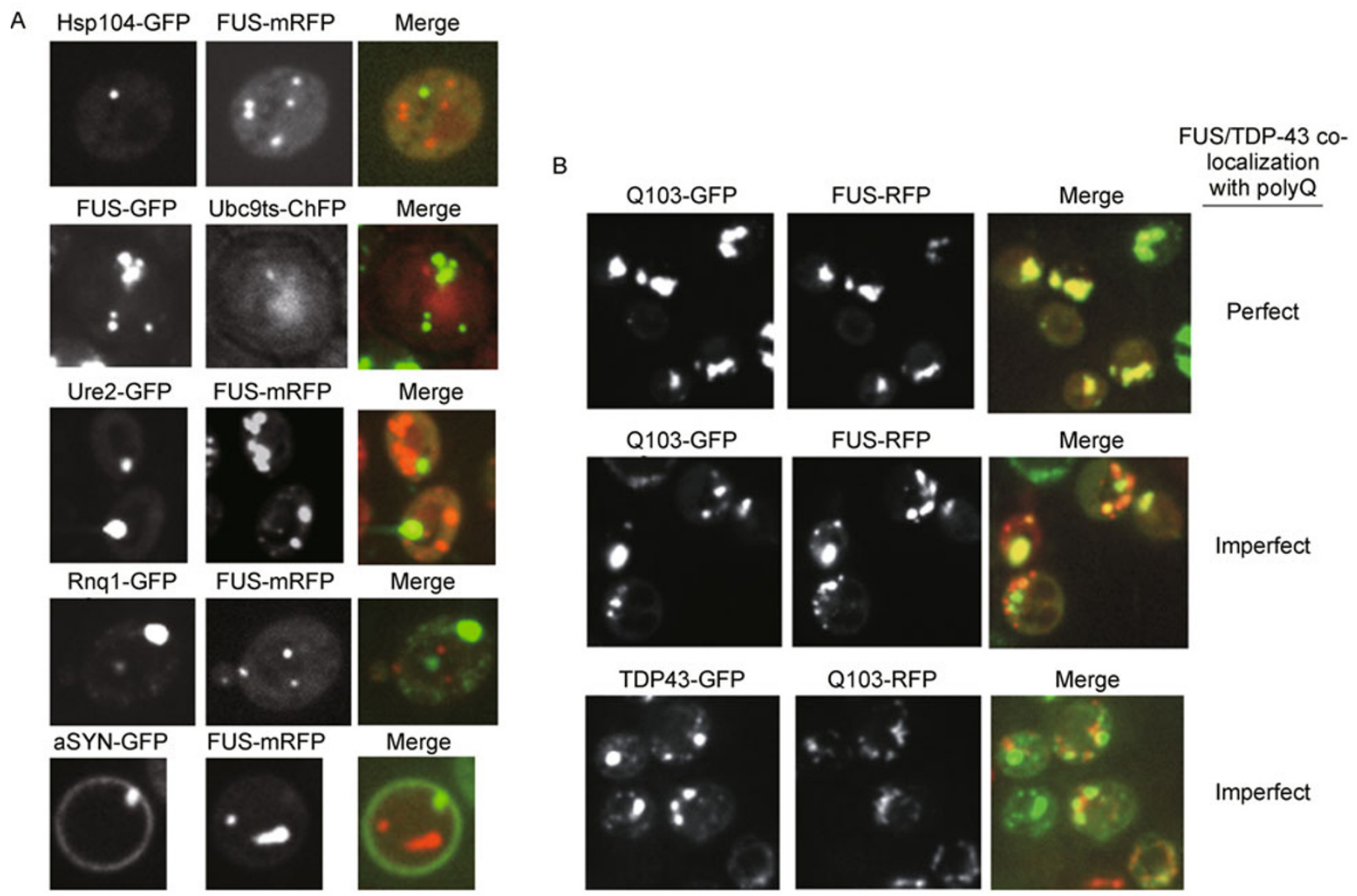

Figure 4. FUS aggregates do not co-localize with several IPOD markers. (A) The GAL1-controlled fluorescent protein fusions of FUS and several characterized IPOD markers (Hsp104, Ubc9ts, Ure2, Rnq1) (Kaganovich et al., 2008), as well as amyloidogenic a-synuclein (aSYN), were co-expressed and monitored by confocal fluorescence microscopy. (B) The GAL1-controlled FUS-RFP and a polyglutamine tract fused to GFP (HttQ103-GFP) partially co-localize. A similar result was observed for the GAL1-controlled TDP-43-GFP and HttQ103-RFP.

HttQ103 and HttQ25 using the same sedimentation assay (Fig. 6B). Cell extracts were treated with $0.5 \%$ Triton X-100 and spun down. Pellet fractions were treated with $1 \times$ SDSPAGE Sample buffer (see MATERIALS AND METHODS), incubated at room temperature or boiled and then immediately subjected to SDS-PAGE. HttQ103 aggregates showed striking resistance to SDS treatment at room temperatures; protein complexes were stuck in the wells of the $10 \%$ polyacrylamide gel and could be detected inside the gel only after boiling. Similar behavior was reported for native yeast prion aggregates (Speransky et al., 2001; Kryndushkin et al., 2003). Instead, both FUS and TDP43 could mostly enter the gel immediately after brief SDS treatment of the pellet fractions at room temperatures (compare lanes " $P$ " and " $P$ *" in Fig. 6B), indicating a significant difference in the biophysical properties of the aggregates. However, both proteins did show a mild resistance to SDS in the absence of heat and prolonged incubation.

The filter retardation assay is an established method for characterizing misfolded and aggregated proteins in yeast (Johnson et al., 2008; Alberti et al., 2009). We treated cell extracts with $1 \%$ SDS at room temperature for 15 min prior to vacuum blotting them to a cellulose acetate membrane $(0.2 \mu \mathrm{m}$ pore size). While HttQ103 aggregates remained SDS-insoluble and were unable to pass through the membrane, FUS aggregates were much less resistant (Fig. 6C). Similar SDS sensitivity was previously reported for TDP-43 (Johnson et al., 2008).

\section{The yeast prion-like amino terminus of FUS does not propagate as a yeast prion}

The amino-terminal domain of FUS has been noted for its striking resemblance to yeast prion domains (Cushman et al., 2010; Udan and Baloh, 2011). Yeast prion domains are distinct in their richness in uncharged polar residues, paucity of charged residues and their apparent native disorder (Toombs et al., 2010). The first approximately 165 amino acids of FUS are not highly charged, but instead are highly enriched in serine, tyrosine, glycine and glutamine (Fig. 3A), compositionally reminiscent of many yeast prion domains. The fact that FUS forms aggregates in diseased motor neural 


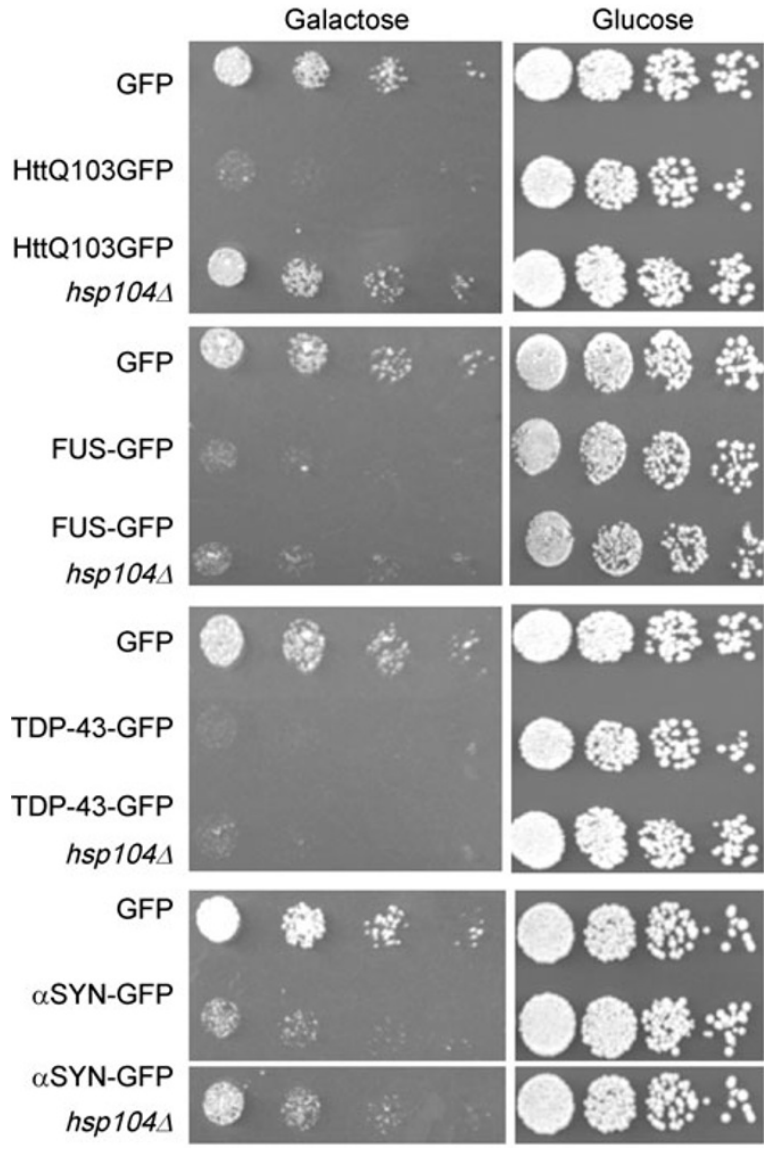

Figure 5. FUS and TDP-43 toxicity is not dependent on Hsp104 function. The toxicities of several aggregation-prone proteins were compared by over-expression using a galactoseinducible promoter in strain 74D-694 containing either wildtype or null HSP104. Cells were grown as described in Fig. 1A.

networks that progressively degenerate appears at least superficially similar to the prion aggregates that propagate vertically through yeast populations.

A yeast Sup35p reporter assay was employed to see if amino-terminal FUS fragments could propagate in two distinct stable states in vivo, as do yeast prions, in which there is a soluble state and a self-propagating amyloid state. Sup35p is a well studied prion protein and is responsible for the $\left[\mathrm{PSI}^{+}\right]$ prion. It contains a portable $\mathrm{N}$-terminal prion domain and a functional C-terminal domain (referred to as Sup35MC), involved in translation termination. When Sup35p is in the prion state, translation is imperfectly terminated at stop codons, resulting in some translational read-through. A premature stop codon in the adenine biosynthesis gene $A D E 2$ is used to report Sup35 function: $\left[P S I^{+}\right]$cells are white/ pink and can grow on media lacking adenine, whereas prionnegative cells are unable to grow on media lacking adenine or grow red on low-adenine media due to the accumulation of an intermediate in the adenine biosynthetic pathway. Fusing
A

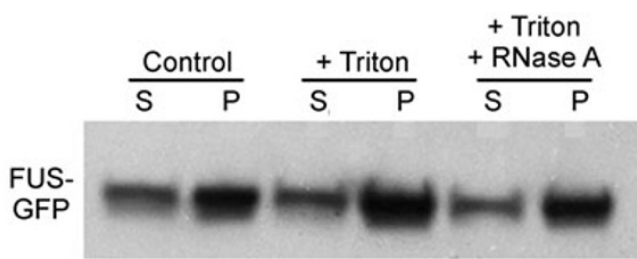

B
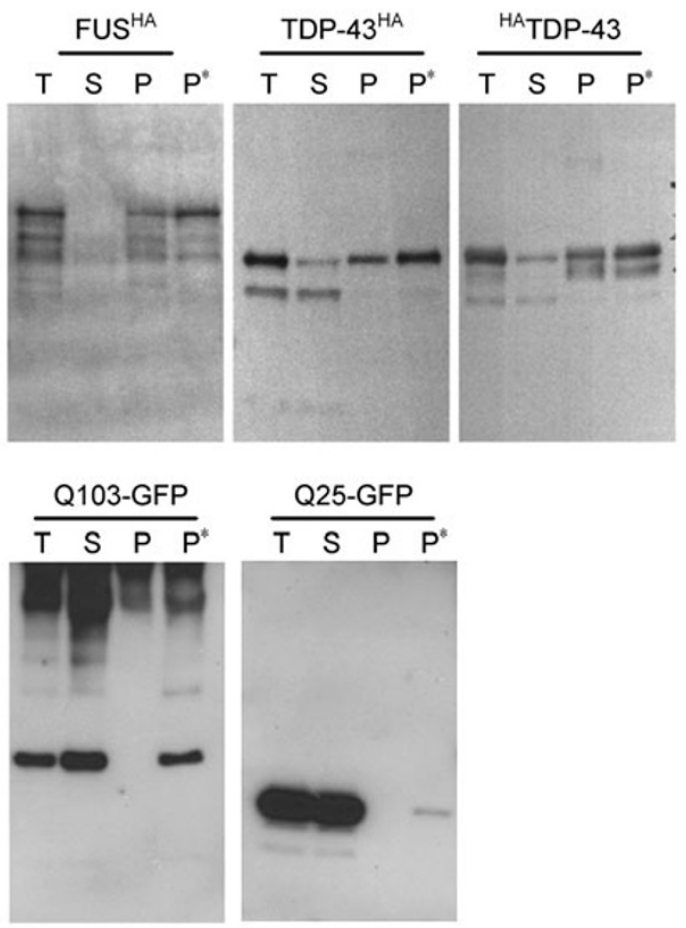

C

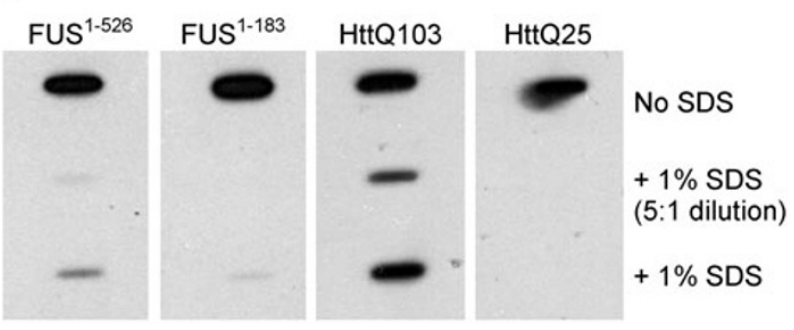

Figure 6. FUS forms high molecular weight SDS-sensitive aggregates. (A) FUS sedimentation does not require association with membranes. Protein lysates were prepared from yeast cells expressing FUS-GFP. Sedimentation of the lysates was performed before or after Triton X-100 and RNase A treatments; $S$, supernatant and $P$, pellet fraction. $(B)$ Lysates from yeast cells expressing FUS-HA, TDP-43-HA, HA-TDP-43, HttQ103GFP or HttQ25-GFP were prepared, sedimented as above and subjected to SDS-PAGE. T, total lysate; $S$, supernatant; P, pellet (no heated incubation); $\mathrm{P}^{*}$, pellet (heated for $5 \mathrm{~min}$ at $96^{\circ} \mathrm{C}$ ). (C) Filter retardation assay. Lysates of cells expressing FUS and polyglutamine GFP fusions were treated with $1 \%$ SDS, vacuum blotted to cellulose acetate $(0.2 \mu \mathrm{m}$ pore size) and immunostained with anti-GFP antibody. 
A



B
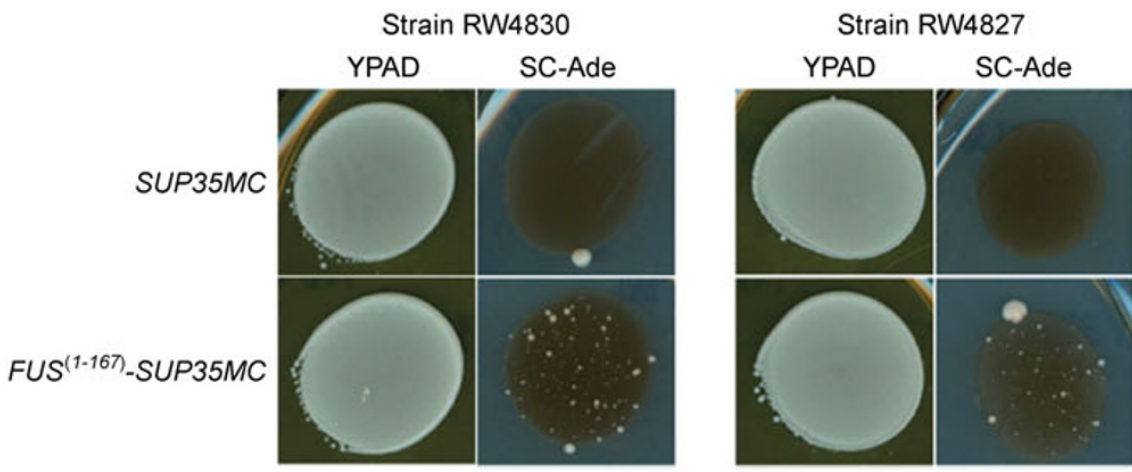

C



Isolate $A 3$

Guanidine-sensitive Ade+ isolates

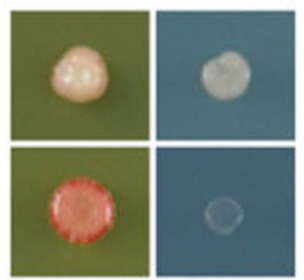

Isolate C5

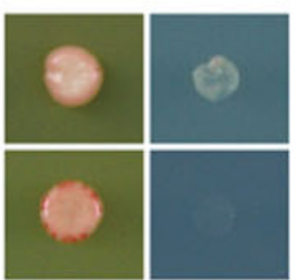

Isolate $D 4$

D

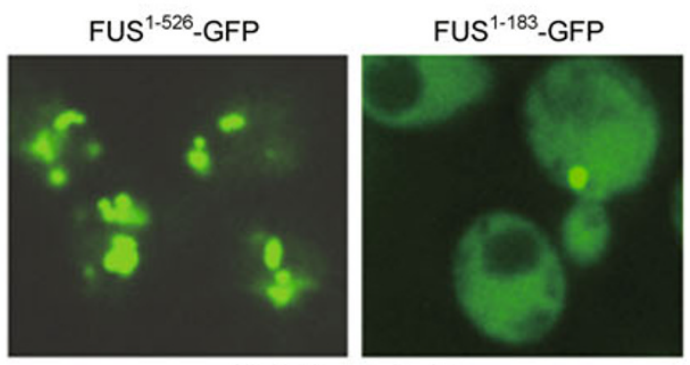

Galactose induction 
protein fragments to Sup35MC, lacking its native aminoterminal prion domain, is an established method for screening for new prion-capable proteins (Chernoff et al., 2000; Kushnirov et al., 2000; Santoso et al., 2000; Alberti et al., 2009).

Plasmids coding FUS ${ }^{(1-167)}$-Sup35MC and FUS ${ }^{(1-134)}$ Sup35MC were plasmid swapped (using 5-FOA) in a sup35 $\triangle$ strain harboring SUP35MC on a URA3 plasmid (Fig. 7A and data not shown). Both FUS fusion constructs could support normal Sup35p function (Fig. 7A), indicating that the constructs were not catastrophic to Sup35p folding, solubility or activity. If the FUS fusions are capable of entering a prion state, this should manifest in the appearance of Ade+ isolates on media lacking adenine. Indeed, upon selection on adenine-minus medium, the fusion constructs yielded far more Ade+ isolates than the SUP35MC control that lacks a prion domain (Fig. 7B). However, when we attempted to induce the formation of prions by transiently over-expressing amino-terminal FUS or full-length FUS in the presence of our FUS ${ }^{(1-167)}$-Sup35MC and FUS ${ }^{(1-134)}$-Sup35MC reporters, we did not see a corresponding increase in Ade+ frequency (data not shown).

Yeast prions are distinguished by their reversible curability. If FUS ${ }^{(1-167)}$-Sup35MC and FUS ${ }^{(1-134)}$-Sup35MC are forming prions, this should be marked by an ability to reverse states and return to the prion-negative form (while retaining the capacity to become a prion again). The conversion to the prion-negative state is greatly facilitated by the inhibition of Hsp104p function, either by gene deletion or a chemical inhibitor (millimolar guanidine), which is necessary for the propagation of most amyloid-based yeast prions. In our hands, a small minority of the Ade+ isolates appeared to lose the Ade+ phenotype after growth on millimolar guanidine (Fig. 7C). However, we could not confirm a true reversion by making the HSP104 deletion in some clones, indicating that the reversion of the phenotype might be because of instability and differential growth rates on medium with guanidine. Furthermore, if the fusion proteins are truly able to stably propagate as prions, they should be able to infect other cells with the prion in a cytoplasmic mixing experiment, assuming the recipient is coding a similar FUS-Sup35MC fusion. We were unable to transfer the Ade+ state from any of our Ade+ isolates to recipient reporters (data not shown). Finally, the ability of a specific prion amyloid to recruit GFP fusion proteins (consisting of GFP fused to the prion protein of interest) is an established method for reporting the physical state of prion proteins within a yeast cell. The aggregation levels of FUS ${ }^{(1-183)}$-GFP (CUP1 promoter) expressed in the Ade + cells versus the control Ade- cells were compared. We could not detect any visible aggregation of FUS ${ }^{(1-183)}$-GFP in either case (data not shown and Fig. 7D).

Finally, the aggregation propensity of the FUS N-terminal fragment (amino acids 1-183 fused to GFP) was explored by fluorescent microscopy and filter retardation assay. A robust induction of the FUS ${ }^{(1-183)}$-GFP expression under the GAL1 promoter resulted only in subtle aggregate formation-one aggregate per cell in $50 \%$ of the cells together with intensive cytoplasmic distribution (Fig. 7D). In contrast, under the same expression conditions, full-length FUS exhibited severe aggregation (Fig. 7D). Also, the filter retardation assay showed SDS sensitivity of the FUS ${ }^{(1-183)}$-GFP aggregates (Fig. 6C), whereas SDS resistance is characteristically observed for prion amyloid aggregates. The fundamental differences observed between aggregation of FUS N-terminal fragments and yeast prion-forming proteins argue against this domain alone conferring a prion capacity of FUS in yeast.

\section{DISCUSSION}

Protein misfolding and aggregation are a pathological hallmark of several neurodegenerative diseases, including Alzheimer's disease, Huntington's disease and amyotrophic lateral sclerosis. Recently the FUS/TLS protein was identified as a new component of the cellular inclusions in non-SOD1 type ALS, atypical fronto-temporal lobar degeneration (FTLD)-U and neuronal intermediate filament inclusion disease (dementia) (NIFID) (Neumann et al., 2009; Deng et al., 2010; Woulfe et al., 2010), indicating that these disorders may share a common pathogenic pathway that ultimately leads to neuronal cell death. To find potentially conserved mechanisms that result in (or from) abnormal FUS aggregation, we utilized a yeast model system that has advantages over mammalian cell lines due to its tractability. By expressing FUS in yeast cells, we were able to recapitulate some of the characteristic features observed in diseased tissue, such as formation of FUS cytoplasmic inclusions and specific co-aggregation with TDP-43 (Deng et al., 2010). Importantly, our model shows that a higher level of FUS expression leads to more severe aggregation and cellular toxicity, suggesting that abnormal cytoplasmic

Figure 7. The SQGY-rich amino terminus of FUS could not form a prion. (A) FUS ${ }^{1-167}$-Sup35MC fusion is functional. A cartoon illustrates how strains RW4827 and RW4830 were transformed. Plasmid swaps were performed in which the LEU2 plasmids with Sup35MC, FUS ${ }^{1-167}$-Sup35MC or empty control were each substituted for $\mathrm{p} 1216$ by 5-FOA selection. Only plasmids coding functional Sup35 permit growth on 5-FOA under this selection. (B) Expression of FUS ${ }^{1-167}$-Sup35MC in the strains RW4827 and RW4830 yielded more Ade+ colonies (prion candidates) than expression of Sup35MC. (C) Some candidates had unstable Ade+ phenotypes, which were exacerbated by the inhibition of Hsp104 function by growth on millimolar guanidine. (D) High-level GAL1controlled expression of the FUS ${ }^{1-183}$-GFP results in less aggregation compared to full-length FUS-GFP as judged by fluorescence microscopy. 
accumulation of FUS in neurons may have a similar consequence and correlate with disease progression.

A particularly interesting conclusion that comes from our work is a striking similarity in FUS and TDP-43 behavior in yeast cells. Both proteins are forming toxic SDS-soluble aggregates that differ biophysically from polyglutamine and amyloid-based yeast prion aggregates (Fig. 6). Furthermore, they show perfect co-aggregation during simultaneous expression in yeast cells, and their interaction was corroborated by co-immunoprecipitation (Fig. 2C and 2D). This interaction is specific since FUS (and TDP-43) aggregates did not co-localize with overproduced amyloidogenic RNQ-GFP, Ure2-GFP or aSYN-GFP proteins, each of which also forms cytoplasmic inclusions in yeast cells (Fig. 4A). Taken together, our results strongly indicate that the mechanism(s) of cellular toxicity for FUS and TDP-43 might be similar.

Both FUS and TDP-43 are involved in formation of stress granules in mammalian cells (Colombrita et al., 2009; Gal et al., 2010; Ito et al., 2011) that may be directly connected with their normal function. Yeast cells do not have close homologues of FUS and TDP-43, but they do have a similar set of proteins that form stress granules under glucose deprivation or elevated temperature (Buchan et al., 2008). Among them are Pab1p, Pub1p, Ngr1p and Pbp1p (yeast orthologues of mammalian PABP, TIA-1, TIA-R and Ataxin-2), all of which are important components of mammalian stress granules (Nonhoff et al., 2007; Buchan and Parker, 2009). Interestingly, Pbp1p was found recently to modify TDP-43 toxicity in yeast and Drosophila (Elden et al., 2010). It would be of interest to more thoroughly investigate the possible interactions between FUS, TDP-43 and stress granule components; perhaps such an approach could contribute to a better understanding of FUS and TDP-43 cellular functions. Many stress granule-related proteins, including FUS and TDP-43, appear to share both a glutamine-rich domain as well as an RNA-binding domain(s). Glutamine-rich domains not only have a strong tendency to self-aggregate, but are likely involved in the assembly process of large stress granule complexes.

A close association of FUS and TDP-43 with expanded polyglutamines deserves further investigation. FUS was identified to be a major component of nuclear polyglutamine aggregates in a Neuro2a cell line (Doi et al., 2008), and likewise TDP-43 is sequestered in polyglutamine aggregates, resulting in the depletion of TDP-43 from the nucleus and the formation of detergent-resistant inclusions (Fuentealba et al., 2010). Interestingly, Ataxin-2 intermediate-length polyglutamine expansions were found more frequently in ALS patients (Elden et al., 2010). Ataxin-2 was found to be associated with TDP-43 under some conditions and to be able to modify TDP43 toxicity. We observed frequent co-aggregation of FUS or TDP-43 with polyglutamine aggregates (Fig. 4B), further supporting a potential link between these RNA-binding proteins and polyglutamine disorders.
We tested the ability of the N-terminal fragment of FUS to serve as a prion in yeast cells. We focused on this fragment because it has high compositional similarity to yeast prion domains (Toombs et al., 2010), which are highly enriched in hydrophilic amino acids like glutamine and asparagine. Such prion domains can adopt an infectious amyloid conformation that propagates by recruiting identical protein sequences into the amyloid aggregate via a template mechanism. Because FUS forms aggregates in neurons, we hypothesized that it may similarly form aggregates in yeast cells by a mechanism similar to the yeast prions. However, we were unable to find evidence that the prion-like domain of FUS has such behavior in yeast cells. In fact, we think that the ability to substitute a native prion domain and drive prion formation may require strong amyloid-forming propensity that appears to be absent in the case of FUS. Because of the fundamental differences observed between FUS aggregation and prion-forming proteins, it appears unlikely that FUS has a pathological mechanism of propagation that is directly reminiscent of prion amyloids. However, it is possible that by utilizing a different approach and/or using different FUS fragments one will be able to isolate an infectious FUS prion. We cannot exclude the possibility that the full-length protein or its sub-domains could form a prion.

During the preparation of this manuscript, Fushimi et al. presented a similar model of FUS proteinopathy (Fushimi et al., 2011). While many of their conclusions are similar to ours, they concluded that the resistance of FUS inclusions to $0.5 \%$ sarcosyl, but not to $2 \%$ sarcosyl, suggests that FUS may be amyloidogenic in yeast. However, based on our experience (Kryndushkin et al., 2003; Kushnirov et al., 2006), $0.5 \%$ sarcosyl may not be sufficient to dissolve all proteinprotein complexes in yeast cell lysates. Instead, we used $1 \%$ SDS and found FUS aggregates to be of much greater sensitivity than polyglutamine aggregates. Amyloids, including yeast prion amyloids, generally show obvious SDS resistance and will frequently get stuck in the loading wells of polyacrylamide gels (an observation we did not find for FUS). Though Fushimi et al. provide suggestive evidence that FUS aggregation is amyloid-like, the nature of FUS aggregates appears to be distinctly different from what has been observed with many amyloidogenic proteins in yeast. TDP-43 aggregates in yeast were previously reported to be nonamyloid in nature (Johnson et al., 2008), which is consistent with our characterization of FUS aggregates.

We observed that FUS and TDP-43 behavior in yeast recapitulates many of the same observations made in neurons and cell culture. This suggests that a simple eukaryotic model could assist in the elucidation of the mechanisms underlying aggregation and cytotoxicity of FUS and other disease-related/aggregation-prone proteins. Considering that there are currently few options for studying FUS proteinopathy in higher organisms, the yeast model and its amenability to high-throughput screens may prove to be a 
helpful tool in understanding cellular degeneration observed with ALS.

\section{MATERIALS AND METHODS}

\section{Yeast strains, growth conditions and plasmids}

Strains BY4741 (MATa his3 leu2 met15 ura3), W303 (MATa can1100 his3-11,15 leu2-3,112 trp1-1 ura3-1 ade2-1), 74D-694 (MATa ade1-14 his3 leu2 trp1 ura3 [PIN $]$ ) (Chernoff et al., 1995) and 74D$694 \Delta h s p 104:: L E U 2$ (a kind gift of Dr. Susan Liebman) were used for FUS and TDP-43 expression. Strains RW4827 (MATa ade2-1 SUQ5 trp1 kar1-1 his3 leu2 ura3 sup35:kanMX) and RW4830 (MATa ade2-1 SUQ5 leu2 ura3 lys2 kar1 sup35:kanMX trp1) were used in the prion assay (discussed below). Strain BY4741 bearing HSP104GFP in the HSP104 genomic locus was purchased from Invitrogen. Yeast cells were grown at $30^{\circ} \mathrm{C}$ in standard synthetic defined media (SD) containing $2 \%$ glucose, or $2 \%$ raffinose (SDRaf), or $2 \%$ galactose and $1 \%$ raffinose (SDGal) as a carbon source. Only required amino acids were added during cell growth. For protein induction cells were grown overnight in SDRaf medium, adjusted to $\mathrm{OD}_{600}=1$ and then incubated in liquid SDGal medium for $4 \mathrm{~h}$. For toxicity assays, yeast cells were grown overnight in liquid SDRaf until they reached log phase. Cultures were then equalized by $\mathrm{OD}_{600}$, serially diluted, spotted onto solid SDGal medium and grown at $30^{\circ} \mathrm{C}$ for 3 days.

Plasmids used in this study are listed in Table 1. The open reading frames corresponding to wild type FUS or TDP-43 were inserted into yeast expression plasmids, pH392cup (centromeric, URA3 marker, CUP1 promoter), pH396gal (high copy, URA3 marker, GAL1 promoter), pH610 (high copy, TRP1, GAL1 promoter) or pH317gal (high copy, LEU2 marker, GAL1 promoter) either intact or in frame with HA, EGFP or mRFP tags at the carboxyl-termini of the proteins. Full-length TDP-43, FUS and truncation variants were prepared using wild-type cDNA plasmids from Open Biosystems; all constructs were confirmed by DNA sequencing.

\section{Sedimentation and filter retardation assays}

To prepare yeast cell extracts strain BY4741 carrying expression plasmid(s) was grown in liquid SDRaf medium overnight and switched to $S D G a l\left(O D_{600}=1\right)$ for induction of protein expression. Cells were incubated for $4 \mathrm{~h}$, harvested, washed in buffer $\mathrm{A}(25 \mathrm{mmol} / \mathrm{L}$ Tris- $\mathrm{HCl}$, $\mathrm{pH} 7.4,150 \mathrm{mmol} / \mathrm{L} \mathrm{NaCl}, 10 \%$ glycerol, $5 \mathrm{mmol} / \mathrm{L}$ EDTA, $1 \mathrm{mmol} / \mathrm{L}$ dithiothreitol and complete protease inhibitor mixture (Roche Applied Science, Indianapolis, IN) and lysed by vortexing with glass beads in the same buffer. To limit protein degradation, in some cases cells were alternatively disrupted by vortexing with glass beads in Urea Lysis Buffer (10 mmol/L Tris, $\mathrm{pH} 7.5,8 \mathrm{~mol} / \mathrm{L}$ urea). Cell debris was removed by centrifugation at $200 \mathrm{~g}$ for $10 \mathrm{~min}$. Protein was measured with BCA reagent (Pierce, Rockford, IL).

For sedimentation analysis, cell lysate aliquots were treated with $1 \%$ Triton X-100 and/or $0.1 \mathrm{mg} / \mathrm{mL}$ RNase $A$ for $10 \mathrm{~min}$ on ice, spun down at $16,000 \mathrm{~g}$ for $10 \mathrm{~min}$, and afterward the pellet and supernatant were collected and compared by standard SDS-PAGE. Immediately prior to loading the samples on an SDS-PAGE gel, the samples were mixed $3: 1$ with a protein-loading buffer containing $4 \%$ sodium dodecyl sulfate. All fractions were heated for $5 \mathrm{~min}$ at $96^{\circ} \mathrm{C}$, except where noted. Immunoblotting was performed using PVDF membranes with
anti-HA antibody or anti-GFP antibody (Roche Applied Science) and AP-conjugated secondary anti-rat IgG (Promega). Signal detection was performed using CDP-Star detection reagent (Perkin Elmer) and Kodak Biomax chemoluminescence film.

For comparing relative detergent resistance of separated fractions, prior to electrophoresis, each fraction (except one pellet fraction) was adjusted to $1 \%$ sodium dodecyl sulfate in gel loading buffer and heated for $5 \mathrm{~min}$ at $96^{\circ} \mathrm{C}$.

The filter retardation assay using cellulose acetate membranes $(0.2 \mu \mathrm{m}$ pore size) was performed as described previously (Muchowski et al., 2000; Johnson et al., 2008). Lysates of cells expressing FUS and polyglutamine GFP fusions were treated with $1 \%$ SDS, vacuum blotted to cellulose acetate, washed and blotted with anti-GFP.

\section{Co-immunoprecipitation}

The interaction between FUS-HA and TDP-43-GFP was analyzed by immunoprecipitation using the protocol and reagents found in the ProFound ${ }^{\mathrm{TM}}$ HA-Tag IP/Co-IP kit (Pierce). FUS-HA was immunoprecipitated from lysates that were prepared from yeast cells that were mechanically disrupted with glass beads in Non-denaturing Lysis Buffer (TBS pH 7.4, 0.1\% tween, 10\% glycerol, $0.1 \mathrm{mmol} / \mathrm{L} \mathrm{AEBSF}$, $0.5 \mathrm{mmol} / \mathrm{L}$ EDTA, protease inhibitor cocktail (Roche)). Following immunoprecipitation, western blots were performed using an antiGFP primary antibody and an AP-conjugated secondary antibody. Signal detection was performed as described above.

\section{Microscopy}

Spinning disc confocal imaging of live yeast cells expressing the appropriate GFP- and RFP-tagged fusion proteins was performed on a Nikon Eclipse TE2000Umicroscope equipped with a $100 \times$ Plan Apo objective. This system is outfittedwith a Spectral Applied Research LMM5 laser merge module to control the output of four diode lasers (excitation at 405, 491, 561 and $655 \mathrm{~nm}$ ), a Yokogawa CSU10 spinning disk unit and a Hamamatsu C9100-13 EM-CCD camera. Confocal imageswere acquired using IPLab imaging software and processed with ImageJ and Adobe Photoshop CS2. During the colocalization experiments, cells were grown overnight in SDRaf medium and then $2 \%$ galactose was added for the induction of the protein of interest. Cells were typically observed after 2-4 hours of induction. $5 \mu \mathrm{L}$ of $2 \mathrm{mg} / \mathrm{mL}$ Concanavalin A (Sigma) solution was applied to microscope slides to immobilize cells. Representative cells were chosen for figures.

\section{Yeast prion assay}

Strains RW4827 and RW4830 are sup35 null, but are viable because Sup35 function is supplied by plasmid p1216, which codes for the Cterminal functional domain of Sup35. Plasmid swaps were performed in which plasmids pFPS201 and pFPS208 were substituted for $\mathrm{p} 1216$ by 5 -FOA selection. To select for adenine positive prion candidates, cells were plated to synthetic complete medium lacking adenine (SCade). The stability of the adenine positive phenotype was tested by growing cells for 40 generations on $3 \mathrm{mmol} / \mathrm{L}$ guanidine and then returning cells to SC-ade. Cytoplasmic transfer experiments (cytoductions) between strains RW4827 and RW4830 were performed as previously described (Wickner et al., 2006). 
Table 1 Plasmids used in the study

\begin{tabular}{|c|c|c|c|c|}
\hline Plasmid name & Promoter & Marker & Copy \# & Source \\
\hline pH317gal FUS-GFP & GAL1 & LEU2 & high copy & this study \\
\hline pH396gal FUS-mRFP & GAL1 & URA3 & high copy & this study \\
\hline pH317gal TDP43-GFP & GAL1 & LEU2 & high copy & this study \\
\hline pH392c FUS ${ }^{1-526}$-GFP & CUP1 & URA3 & centromeric & this study \\
\hline pH392c FUS ${ }^{1-422}$-GFP & CUP1 & URA3 & centromeric & this study \\
\hline pH392c FUS ${ }^{292-526}$-GFP & CUP1 & URA3 & centromeric & this study \\
\hline pH392c FUS ${ }^{292-422-G F P}$ & CUP1 & URA3 & centromeric & this study \\
\hline p784 Ure2-GFP & URE2 & LEU2 & high copy & this study \\
\hline pH396gal RNQ1-GFP & GAL1 & URA3 & high copy & this study \\
\hline HttQ103-GFP & GAL1 & URA3 & high copy & Meriin et al., 2002 \\
\hline HttQ25-GFP & GAL1 & URA3 & high copy & Meriin et al., 2002 \\
\hline pEsc NLS-tdTomato & GAL1 & LEU2 & high copy & Kaganovich et al., 2008 \\
\hline pEsc CherryFP-Ubc9ts & GAL1 & URA3 & high copy & Kaganovich et al., 2008 \\
\hline pH396gal aSYN-GFP & GAL1 & URA3 & high copy & this study \\
\hline pH396gal FUS ${ }^{1-183}$-GFP & GAL1 & URA3 & high copy & this study \\
\hline pFPS201(FUS ${ }^{(1-167)}$-Sup35MC) & $A D H 1$ & LEU2 & centromeric & this study \\
\hline pFPS208(FUS ${ }^{(1-134)}$-Sup35MC) & $A D H 1$ & LEU2 & centromeric & this study \\
\hline pFPS219(FUS ${ }^{1-526}$ ) & GAL1 & TRP1 & high copy & this study \\
\hline pFPS217(FUS ${ }^{1-134}$ ) & GAL1 & TRP1 & high copy & this study \\
\hline pFPS257(FUS ${ }^{1-253}$ ) & GAL1 & TRP1 & high copy & this study \\
\hline pFPS258(FUS $\left.{ }^{135-526}\right)$ & GAL1 & TRP1 & high copy & this study \\
\hline pFPS258(FUS $\left.{ }^{135-526}\right)$ & GAL1 & TRP1 & high copy & this study \\
\hline pFPS245(FUS $\left.{ }^{254-526}\right)$ & $G A L 1$ & TRP1 & high copy & this study \\
\hline pFPS251(FUS $\left.{ }^{436-526}\right)$ & GAL1 & TRP1 & high copy & this study \\
\hline pFPS264(FUS $\left.{ }^{1-526+H A}\right)$ & GAL1 & TRP1 & high copy & this study \\
\hline pFPS259(FUS $\left.{ }^{135-526+\mathrm{HA}}\right)$ & GAL1 & TRP1 & high copy & this study \\
\hline pFPS256(FUS $\left.{ }^{254-526+H A}\right)$ & GAL1 & $T R P 1$ & high copy & this study \\
\hline pFPS229(FUS ${ }^{(1-183)}+$ GFP) & GAL1 & TRP1 & high copy & this study \\
\hline pFPS266(FUS ${ }^{1-526}$ ) & CUP1 & URA3 & centromeric & this study \\
\hline pFPS270(FUS $\left.{ }^{1-526+H A}\right)$ & CUP1 & URA3 & centromeric & this study \\
\hline pFPS268(FUS ${ }^{254-526+H A}$ ) & CUP1 & URA3 & centromeric & this study \\
\hline pFPS234(TDP-43 + HA) & CUP1 & URA3 & centromeric & this study \\
\hline pFPS235(HA + TDP-43) & CUP1 & URA3 & centromeric & this study \\
\hline p1216 (SUP35C) & $A D H 1$ & URA3 & centromeric & this study \\
\hline p1166 (SUP35MC) & $A D H 1$ & LEU2 & centromeric & this study \\
\hline
\end{tabular}

\section{ACKNOWLEDGEMENTS}

We thank Herman Edskes (NIH) for providing plasmids and for his thoughtful suggestions, Tetsade Piermartiri for her assistance with western blotting, Susan Liebman (University of Illinois Chicago) for providing yeast strains, Kevin O'Connell (NIH) for expert assistance with confocal microscopy, Judith Frydman (Stanford University) and Michael Sherman (Boston University) for plasmids. This research was supported by Uniformed Services University of the Health Sciences and the Intramural Research Program of the National Institutes of Health, National Institute of Diabetes and Digestive and Kidney Diseases.

\section{ABBREVIATIONS}

ALS, amyotrophic lateral sclerosis; FTLD, fronto-temporal lobar degeneration; FTLD-U, FTLD with ubiquitin-positive inclusions; NIFID, neuronal intermediate filament inclusion disease (dementia); 5-FOA, 5-fluoroorotic acid

\section{REFERENCES}

Alberti, S., Halfmann, R., King, O., Kapila, A., and Lindquist, S. (2009). A systematic survey identifies prions and illuminates sequence features of prionogenic proteins. Cell 137, 146-158. 
Ayala, Y.M., Zago, P., D’Ambrogio, A., Xu, Y.F., Petrucelli, L., Buratti, E., and Baralle, F.E. (2008). Structural determinants of the cellular localization and shuttling of TDP-43. J Cell Sci 121, 3778-3785.

Bharadwaj, P., Martins, R., and Macreadie, I. (2010). Yeast as a model for studying Alzheimer's disease. FEMS Yeast Res 10, 961-969.

Bosco, D.A., Lemay, N., Ko, H.K., Zhou, H., Burke, C., Kwiatkowski, T.J. Jr, Sapp, P., McKenna-Yasek, D., Brown, R.H. Jr, and Hayward, L.J. (2010). Mutant FUS proteins that cause amyotrophic lateral sclerosis incorporate into stress granules. Hum Mol Genet 19, 4160-4175.

Braun, R.J., Büttner, S., Ring, J., Kroemer, G., and Madeo, F. (2010). Nervous yeast: modeling neurotoxic cell death. Trends Biochem Sci 35, 135-144.

Buchan, J.R., Muhlrad, D., and Parker, R. (2008). P bodies promote stress granule assembly in Saccharomyces cerevisiae. J Cell Biol 183, 441-455.

Buchan, J.R., and Parker, R. (2009). Eukaryotic stress granules: the ins and outs of translation. Mol Cell 36, 932-941.

Chernoff, Y.O., Galkin, A.P., Lewitin, E., Chernova, T.A., Newnam, G. P., and Belenkiy, S.M. (2000). Evolutionary conservation of prionforming abilities of the yeast Sup35 protein. Mol Microbiol 35, 865-876.

Chernoff, Y.O., Lindquist, S.L., Ono, B., Inge-Vechtomov, S.G., and Liebman, S.W. (1995). Role of the chaperone protein Hsp104 in propagation of the yeast prion-like factor [psi+]. Science 268, 880-884.

Colombrita, C., Zennaro, E., Fallini, C., Weber, M., Sommacal, A., Buratti, E., Silani, V., and Ratti, A. (2009). TDP-43 is recruited to stress granules in conditions of oxidative insult. J Neurochem 111, 1051-1061.

Cooper, A.A., Gitler, A.D., Cashikar, A., Haynes, C.M., Hill, K.J., Bhullar, B., Liu, K., Xu, K., Strathearn, K.E., Liu, F., et al. (2006). Alpha-synuclein blocks ER-Golgi traffic and Rab1 rescues neuron loss in Parkinson's models. Science 313, 324-328.

Cushman, M., Johnson, B.S., King, O.D., Gitler, A.D., and Shorter, J. (2010). Prion-like disorders: blurring the divide between transmissibility and infectivity. J Cell Sci 123, 1191-1201.

Deng, H.X., Zhai, H., Bigio, E.H., Yan, J., Fecto, F., Ajroud, K., Mishra, M., Ajroud-Driss, S., Heller, S., Sufit, R., et al. (2010). FUS-immunoreactive inclusions are a common feature in sporadic and non-SOD1 familial amyotrophic lateral sclerosis. Ann Neurol 67, 739-748.

Doi, H., Okamura, K., Bauer, P.O., Furukawa, Y., Shimizu, H., Kurosawa, M., Machida, Y., Miyazaki, H., Mitsui, K., Kuroiwa, Y., et al. (2008). RNA-binding protein TLS is a major nuclear aggregate-interacting protein in huntingtin exon 1 with expanded polyglutamine-expressing cells. J Biol Chem 283, 6489-6500.

Duennwald, M.L., and Lindquist, S. (2008). Impaired ERAD and ER stress are early and specific events in polyglutamine toxicity. Genes Dev 22, 3308-3319.

Elden, A.C., Kim, H.J., Hart, M.P., Chen-Plotkin, A.S., Johnson, B.S., Fang, X., Armakola, M., Geser, F., Greene, R., Lu, M.M., et al. (2010). Ataxin-2 intermediate-length polyglutamine expansions are associated with increased risk for ALS. Nature 466, 1069-1075.

Fuentealba, R.A., Udan, M., Bell, S., Wegorzewska, I., Shao, J., Diamond, M.I., Weihl, C.C., and Baloh, R.H. (2010). Interaction with polyglutamine aggregates reveals a $\mathrm{Q} / \mathrm{N}$-rich domain in TDP43. J Biol Chem 285, 26304-26314.

Fushimi, K., Long, C., Jayaram, N., Chen, X., Li, L., and Wu, J.Y. (2011). Expression of human FUS/TLS in yeast leads to protein aggregation and cytotoxicity, recapitulating key features of FUS proteinopathy. Protein Cell 2, 141-149

Gal, J., Zhang, J., Kwinter, D.M., Zhai, J., Jia, H., Jia, J., and Zhu, H. (2010). Nuclear localization sequence of FUS and induction of stress granules by ALS mutants. Neurobiol Aging. Jul 29. [Epub ahead of print]. doi:10.1016/ j.neurobiolaging.2010.06.010.

Iko, Y., Kodama, T.S., Kasai, N., Oyama, T., Morita, E.H., Muto, T., Okumura, M., Fujii, R., Takumi, T., Tate, S., et al. (2004). Domain architectures and characterization of an RNA-binding protein, TLS. J Biol Chem 279, 44834-44840.

Ito, D., Seki, M., Tsunoda, Y., Uchiyama, H., and Suzuki, N. (2011). Nuclear transport impairment of amyotrophic lateral sclerosislinked mutations in FUS/TLS. Ann Neurol 69, 152-162.

Johnson, B.S., McCaffery, J.M., Lindquist, S., and Gitler, A.D. (2008). A yeast TDP-43 proteinopathy model: Exploring the molecular determinants of TDP-43 aggregation and cellular toxicity. Proc Natl Acad Sci U S A 105, 6439-6444.

Kaganovich, D., Kopito, R., and Frydman, J. (2008). Misfolded proteins partition between two distinct quality control compartments. Nature 454, 1088-1095.

Khurana, V., and Lindquist, S. (2010). Modelling neurodegeneration in Saccharomyces cerevisiae: why cook with baker's yeast? Nat Rev Neurosci 11, 436-449.

Kim, S.H., Shanware, N.P., Bowler, M.J., and Tibbetts, R.S. (2010). Amyotrophic lateral sclerosis-associated proteins TDP-43 and FUS/TLS function in a common biochemical complex to coregulate HDAC6 mRNA. J Biol Chem 285, 34097-34105.

Krobitsch, S., and Lindquist, S. (2000). Aggregation of huntingtin in yeast varies with the length of the polyglutamine expansion and the expression of chaperone proteins. Proc Natl Acad Sci U S A 97, 1589-1594.

Kryndushkin, D.S., Alexandrov, I.M., Ter-Avanesyan, M.D., and Kushnirov, V.V. (2003). Yeast [PSI+] prion aggregates are formed by small Sup35 polymers fragmented by Hsp104. J Biol Chem 278 , 49636-49643.

Kushnirov, V.V., Alexandrov, I.M., Mitkevich, O.V., Shkundina, I.S., and Ter-Avanesyan, M.D. (2006). Purification and analysis of prion and amyloid aggregates. Methods 39, 50-55.

Kushnirov, V.V., Kochneva-Pervukhova, N.V., Chechenova, M.B., Frolova, N.S., and Ter-Avanesyan, M.D. (2000). Prion properties of the Sup35 protein of yeast Pichia methanolica. EMBO J 19 , 324-331.

Kwiatkowski, T.J. Jr, Bosco, D.A., Leclerc, A.L., Tamrazian, E., Vanderburg, C.R., Russ, C., Davis, A., Gilchrist, J., Kasarskis, E. J., Munsat, T., et al. (2009). Mutations in the FUS/TLS gene on chromosome 16 cause familial amyotrophic lateral sclerosis. Science 323, 1205-1208.

Lagier-Tourenne, C., Polymenidou, M., and Cleveland, D.W. (2010). TDP-43 and FUS/TLS: emerging roles in RNA processing and neurodegeneration. Hum Mol Genet 19, R46-R64.

Ling, S.C., Albuquerque, C.P., Han, J.S., Lagier-Tourenne, C., Tokunaga, S., Zhou, H., and Cleveland, D.W. (2010). ALSassociated mutations in TDP-43 increase its stability and promote TDP-43 complexes with FUS/TLS. Proc Natl Acad Sci U S A 107, 
13318-13323.

McGlinchey, R., Kryndushkin, D., and Wickner, R.B. (2011). Suicidal $[\mathrm{PSI}+]$ is a lethal yeast prion. Proc Natl Acad Sci U S A. (In press)

Meriin, A.B., Zhang, X., He, X., Newnam, G.P., Chernoff, Y.O., and Sherman, M.Y. (2002). Huntington toxicity in yeast model depends on polyglutamine aggregation mediated by a prion-like protein Rnq1. J Cell Biol 157, 997-1004.

Meriin, A.B., Zhang, X., Miliaras, N.B., Kazantsev, A., Chernoff, Y.O., McCaffery, J.M., Wendland, B., and Sherman, M.Y. (2003). Aggregation of expanded polyglutamine domain in yeast leads to defects in endocytosis. Mol Cell Biol 23, 7554-7565.

Muchowski, P.J., Schaffar, G., Sittler, A., Wanker, E.E., Hayer-Hartl, M.K., and Hartl, F.U. (2000). Hsp70 and hsp40 chaperones can inhibit self-assembly of polyglutamine proteins into amyloid-like fibrils. Proc Natl Acad Sci U S A 97, 7841-7846.

Neumann, M., Roeber, S., Kretzschmar, H.A., Rademakers, R., Baker, M., and Mackenzie, I.R. (2009). Abundant FUS-immunoreactive pathology in neuronal intermediate filament inclusion disease. Acta Neuropathol 118, 605-616.

Nonhoff, U., Ralser, M., Welzel, F., Piccini, I., Balzereit, D., Yaspo, M. L., Lehrach, H., and Krobitsch, S. (2007). Ataxin-2 interacts with the DEAD/H-box RNA helicase DDX6 and interferes with P-bodies and stress granules. Mol Biol Cell 18, 1385-1396.

Outeiro, T.F., and Lindquist, S. (2003). Yeast cells provide insight into alpha-synuclein biology and pathobiology. Science 302, 1772-1775.

Rosen, D.R., Siddique, T., Patterson, D., Figlewicz, D.A., Sapp, P., Hentati, A., Donaldson, D., Goto, J., O'Regan, J.P., Deng, H.X., et al. (1993). Mutations in Cu/Zn superoxide dismutase gene are associated with familial amyotrophic lateral sclerosis. Nature 362 , $59-62$.

Salnikova, A.B., Kryndushkin, D.S., Smirnov, V.N., Kushnirov, V.V., and Ter-Avanesyan, M.D. (2005). Nonsense suppression in yeast cells overproducing Sup35 (eRF3) is caused by its non-heritable amyloids. J Biol Chem 280, 8808-8812.

Santoso, A., Chien, P., Osherovich, L.Z., and Weissman, J.S. (2000). Molecular basis of a yeast prion species barrier. Cell 100, 277-288.

Serio, T.R., Cashikar, A.G., Kowal, A.S., Sawicki, G.J., Moslehi, J.J., Serpell, L., Arnsdorf, M.F., and Lindquist, S.L. (2000). Nucleated conformational conversion and the replication of conformational information by a prion determinant. Science 289, 1317-1321.

Sharma, N., Brandis, K.A., Herrera, S.K., Johnson, B.E., Vaidya, T., Shrestha, R., and Debburman, S.K. (2006). alpha-Synuclein budding yeast model: toxicity enhanced by impaired proteasome and oxidative stress. J Mol Neurosci 28, 161-178.

Speransky, V.V., Taylor, K.L., Edskes, H.K., Wickner, R.B., and Steven, A.C. (2001). Prion filament networks in [URE3] cells of Saccharomyces cerevisiae. J Cell Biol 153, 1327-1336.

Sreedharan, J., Blair, I.P., Tripathi, V.B., Hu, X., Vance, C., Rogelj, B., Ackerley, S., Durnall, J.C., Williams, K.L., Buratti, E., et al. (2008). TDP-43 mutations in familial and sporadic amyotrophic lateral sclerosis. Science 319, 1668-1672.

Toombs, J.A., McCarty, B.R., and Ross, E.D. (2010). Compositional determinants of prion formation in yeast. Mol Cell Biol 30, 319-332.

Udan, M., and Baloh, R.H. (2011). Implications of the prion-related Q/ $\mathrm{N}$ domains in TDP-43 and FUS. Prion 5, 1-5.

Vance, C., Rogelj, B., Hortobágyi, T., De Vos, K.J., Nishimura, A.L., Sreedharan, J., Hu, X., Smith, B., Ruddy, D., Wright, P., et al. (2009). Mutations in FUS, an RNA processing protein, cause familial amyotrophic lateral sclerosis type 6. Science 323, 1208-1211.

Wickner, R.B., Edskes, H.K., and Shewmaker, F. (2006). How to find a prion: [URE3], [PSI+] and [beta]. [beta] Methods 39, 3-8.

Wickner, R.B., Shewmaker, F., Edskes, H., Kryndushkin, D., Nemecek, J., McGlinchey, R., Bateman, D., and Winchester, C. L. (2010). Prion amyloid structure explains templating: how proteins can be genes. FEMS Yeast Res 10, 980-991.

Woulfe, J., Gray, D.A., and Mackenzie, I.R. (2010). FUS-immunoreactive intranuclear inclusions in neurodegenerative disease. Brain Pathol 20, 589-597.

Yeger-Lotem, E., Riva, L., Su, L.J., Gitler, A.D., Cashikar, A.G., King, O.D., Auluck, P.K., Geddie, M.L., Valastyan, J.S., Karger, D.R., et al. (2009). Bridging high-throughput genetic and transcriptional data reveals cellular responses to alpha-synuclein toxicity. Nat Genet 41, 316-323.

Zinszner, H., Sok, J., Immanuel, D., Yin, Y., and Ron, D. (1997). TLS (FUS) binds RNA in vivo and engages in nucleo-cytoplasmic shuttling. J Cell Sci 110, 1741-1750. 